\title{
Ontology-Based Structured Cosine Similarity in Speech Document Summarization
}

\author{
Soe-Tsyr Yuan ${ }^{1}$ and Jerry Sun $^{2}$ \\ ${ }^{1}$ Dept. of Management Information System \\ National Chengchi University, Taipei, Taiwan \\ ${ }^{2}$ Dept. of Information Management \\ Fu-Jen University, Taiwan
}

\begin{abstract}
Development of algorithms for automated text categorization in massive text document sets is an important research area of data mining and knowledge discovery. Most of the text-clustering methods were grounded in the term-based measurement of distance or similarity, ignoring the structure of terms in documents. In this paper we present a novel method named Structured Cosine Similarity that furnishes document clustering with a new way of modeling on document summarization, considering the structure of terms in documents in order to improve the quality of speech document clustering.
\end{abstract}

\section{Introduction}

Development of algorithms for automated text categorization in massive text document sets is an important research area of data mining and knowledge discovery. Most of the text- clustering methods were grounded in the term-based measurement of distance or similarity, ignoring the structure of terms in documents. In this paper we present a novel method named Structured Cosine Similarity that furnishes text clustering with a new way of modeling on document summarization, considering the structure of terms in documents in order to improve the quality of clustering. This study was motivated by the desired high performance of clustering speech documents attained from the wireless experience oral sharing conducted by mobile workforce of enterprises, fulfilling audio based knowledge management. In other words, this problem aims to facilitate knowledge acquisition and sharing by speech.

In spite of the advent of the ASR technology's growing maturity, speech documents attained are highly ill structured, rendering successive knowledge categorization and distribution difficult. Knowledge categorization, in general, involves document summarization and summarization clustering [1].

Most of existing methods about document summarization rest on translation of documents into term vectors. For instance, the tfidf method [2] summarized documents with term-frequency inverse document. In this method, term frequency $t_{j i}$ is the number of times word $i$ occurs in document $j$; the inverse document frequency $g_{i}$ is low if it occurs in many documents and is highest if the word occurs in only one ${ }^{1}$; a document vector is then

${ }^{1}$ The underlying assumptions of this weighting scheme are based on two empirical observations regarding text. First, the more times a term appears in a document, the more likely it is that term represented as a vector of $S_{j i}=t_{j i} * g_{i}$. Another improved method on text summarization is Latent Semantic Indexing (LSI) [3, 4, 5] that further decomposed a term document matrix (resulted from the tfidf method) using a technique called singular value decomposition to consider documents that have many words in common to be semantically close, and ones with few words in common to be semantically distant.

When addressing document clustering, clustering with the complex LSI-based summarization correlates surprisingly well with how a human being, looking at documents, might classify a document collection, but suffers from heavy computation overhead [6]. Fortunately, with the simple tfidf-based summarization Spherical K-Means Clustering (SKC) [7, 8] (evolved from the simple clustering method of K-Means [9]) was proved to outperform LSI in terms of exhibiting the same level of clustering quality as LSI but with a significance improvement in efficiency.

During clustering, term vectors are clustered with certain similarity measures, such as Euclidean, Cosine, Extended Jaccard, etc. For instance, LSI employed Euclidean similarity, while SKC exerted Cosine similarity. [10] showed that Euclidean similarity is translation invariant but scale sensitive while Cosine similarity is translation sensitive but scale invariant and that the Extended Jaccard has aspects of both properties. If clusters are to be meaningful, the similarity measure should be invariant to transformations natural to the problem domain. Furthermore, normalization may strongly affect clustering in a positive or negative way. Accordingly, a good similarity measure has to reflect the underlying semantics for the given task.

With the task of text clustering, Cosine similarity is a simple measure endows documents with the same composition but different sizes to be treated identically which makes this the most popular measure for clustering text documents. Moreover, due to this property, term vectors can be normalized to the unit sphere for more efficient processing as conducted in $[7,8]$.

As far as we have discussed, the SKC method is comparably highly regarded due to its simplicity and good quality. However, it naturally comes to a question if there is room for further improvement that can be rendered to the SKC method so as to advance the clustering quality? This improvement is exceedingly important especially for audio-based knowledge management among mobile work force. The rationale is as follows: higher quality of performance render mobile workforce with handset devices (that are limited with resources as power, memory,

is relevant to the topic of the document. Second, the more times the term occurs throughout all document, the more poorly the term discriminates between documents. 
screen, etc.) less overhead in sharing and accessing experience and knowledge, especially for time critical tasks requiring decisions of quick response.

Examining the SKC method (and most of existing text clustering algorithms), we discover they disregard whatsoever (semi) structured relations between terms in a document (regardless of the methods employed to attain the terms) ${ }^{2}$. Accordingly, one way of exploring the advancement of the SKC method is to utilize these structured relations.

In this paper, we present a novel methodology named Structured Cosine Similarity that furnishes text clustering with a new way of modeling on document summarization by considering the structure of terms in documents in order to improve the clustering quality. The evaluation results show this new modeling does advance the SKC method in the respects of quality, stability and efficiency.

The remainder of this paper is organized as follows: Section 2 presents the Structured Cosine Similarity methodology. A comprehensive evaluation is then conducted and described in Section 3. Finally, Section 4 concludes this paper with future fruitful research.

\section{Structured Cosine Similarity (SCS)} follows:

The main ideas (assumptions) underlying SCS are as

- There exists a certain knowledge domain in which the task of knowledge categorization is undertaken. This knowledge domain is presumed to be task-oriented and can be modeled by a task-oriented ontology. We currently exert as the knowledge domain troubleshooting of electronics (e.g., color laser printers, digital cameras, etc.) In the application of audio based knowledge management, simply by voice mobile workforce of electronic troubleshooting are able to share and access troubleshooting experience that is primarily underlined by certain relevant troubleshooting categorization (accordingly modeled by an electronictroubleshooting ontology).

- The ASR technique employed to generate speech documents of audio queries is discrete word recognition that recognizes those key words appearing in the nodes of the task-oriented ontology. In our implementation IBM ViaVoice [11] is exercised to generate speech documents.

- The task-oriented ontology serves as the structure employed in modeling the new way of document summarization so as to advance the performance of the SKC method.

In the following subsections, SCS is described at two-fold: (1) the task-oriented ontology employed in this research (2) the SCS method.

\subsection{Task-Oriented Ontology}

In the context of knowledge sharing, the term ontology means a specification of a conceptualization. That is, an ontology is a description of the concepts and relationships that can exist for

\footnotetext{
${ }^{2}$ Structures utilized in text mining mostly were seen as adaptive ranking of web pages according to their link structures or explanation provision of generated text clusters according to relevant concept taxonomies.
}

an agent or a community of agents, and can be designed for the purpose of enabling knowledge sharing and reuse [12].

A task-oriented ontology specifies concepts and relationships relevant to a particular task such as the task of electron troubleshooting (in which knowledge and experience are often related with other by causal relationships). Figure 1 shows a fragmented exemplar of such task-oriented ontology. Nodes and links in a given task-oriented ontology are defined as follows:

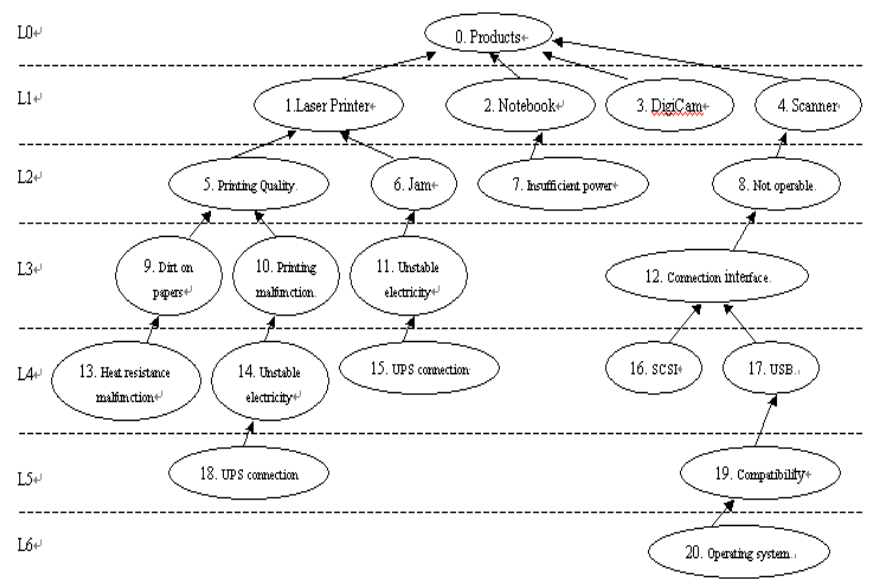

Figure 1. A fragmented exemplar of task-oriented ontology.

Nodes: a concept in the domain of the task. Nodes of higher levels are the causes more abstract than those of lower levels. For instance, node 11 of "unstable electricity" (a cause of node 6 of "paper jam") is a cause that is more abstract than node 15 of "UPS connection problem".

- Links: Links are directional, indicating the directions of the causal relationships (in which the heads of the links represent causes while the tails denote consequences). For instance, the cause denoted by node 15 of "UPS connection problem" often results in the consequence of node 11 of "unstable electricity". For simplicity ${ }^{3}$, a cause for multiple consequences is represented by multiple causal links. For instance, the cause of "unstable electricity" is contributable to two consequences of "paper jam" and "printing malfunction", and thus there exist two causal links denoting this situation (a link connecting from node 11 of "unstable electricity" to node 6 of "paper jam" and another link from node 14 of "unstable electricity" to node of "printing malfunction"). On the other hand, multiple causes for a single consequence (if any) are simply modeled by a causal link denoting a causal relationship between the aggregated cause and the consequence (i.e., this aggregated link either can be further refined to multiple independent links between the causes and the refined consequences or conveys that the consequence can be resulted from any cause taking from this aggregated set of causes).

\subsection{The SCS Method}

SCS aims to utilize a given task-oriented ontology to facilitate document summarization and thus involves translation of

${ }^{3}$ In this paper, a task-oriented ontology is modeled as a tree structure between nodes with links. 
documents into term vectors and making the resulting term vectors in accord with the underlying semantics before clustering taking place. This section models SCS document translation using the aforementioned exemplified task-oriented ontology (as shown in Figure 1). To simplify the presentation, Figure 1 is abbreviated as Figure 2 (in which nodes are numbered ${ }^{4}$ level by level from top to bottom except the root).

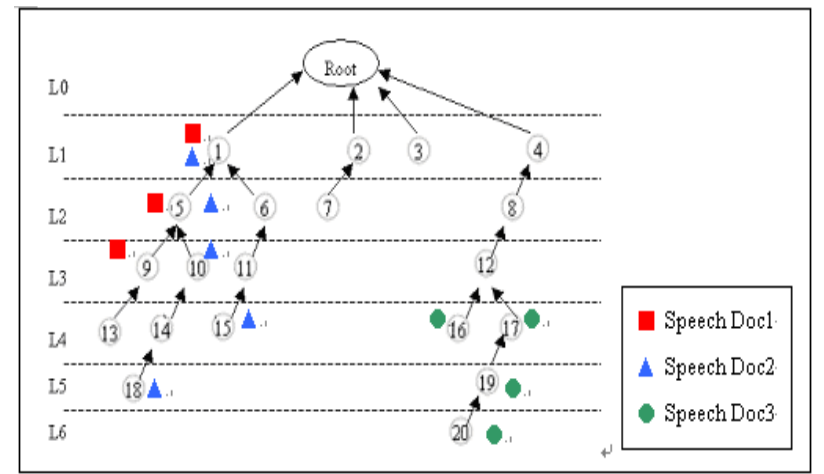

Figure 2. A simplified representation of the task-oriented ontology shown in Figure 1.

After the ASR processing of an audio sharing (query), the resulting speech document originally is a set of words without any structures implied between them. However, these words can be endowed with a structure in the following approach:

- For a given task-oriented ontology, assume there are $m$ nodes (e.g., 20 nodes as shown in Figure 2). Therefore, any speech document $\left(x_{i}, i=1,2, \cdots, n\right)$ is to be represented as a sized-20 vector of $x_{i p}(p=0,1,2, \cdots, m)$. This vector is initialized with a vector of zeros. The value of $x_{i p}$ is increased by 1 when encountering an existence of the $p$ numbered concept node in the speech document. For instance, the term vector of a speech document (containing only node 20) unfolds as $(0,0,0,0,0,0,0,0,0,0,0,0,0,0,0,0,0,0,0,1)$.

- When imposing the structure (implied by the ontology) into a vector, all of the parent nodes of 1-valued $x_{i p}$ are accounted as existence (and thus the resulting vector contains more none-zero term values). Using the last example of document of node 20 , the resulting vector becomes $(0,0,0,1,0,0,0,1,0,0,0,1,0,0,0,0,1,0,1,1)$ as the parent nodes of node 20 include node 4, 8, 12, 17, 19, 20. Afterward, we name this method of imposing structure as "structured" imposing.

- Alternative way of imposing the structure into a vector combines the ideas of tfidf and structured imposing:

- The term frequencies (representing the number of times words occur in document) used in the tfidf

${ }^{4}$ This numbering is simply for uniquely identifying the nodes. Different numberings works as long as the nodes can be uniquely identified. method are replaced with the numbers attained from the method of structured imposing.

- The inverse document frequencies are kept as the constant of 1 to avoid the situation that words on the same path will aggrandize the accounting of their parents ${ }^{5}$ and thus result in smaller values in the inverse document frequencies (loosing the attempted intention of imposing structure between words). Afterward, we will name this approach as s-txn.

The aforementioned idea is then stated in the s-txn formula below:

\section{S-txn formula:}

Term value for the $\mathrm{p}$-numbered concept $=x_{i p} * g_{p} * s_{p}$,

$x_{i p}$ : a number resulting from the approach of structured imposing (applied to the document $x_{i}$ ) for the p-numbered concept node of the task-oriented ontology, $g_{p}=1$, $p=0,1,2, \cdots, m$.

$s_{i}=\left(\sum_{p=1}^{m}\left(x_{i p} g_{p}\right)^{2}\right)^{-1 / 2}$, normalization component.

- Cosine similarity will be exerted to measure the proximity between term vectors owing to its scale invariant (that is addressed in Section 1) and it suitability in comparing high dimensional but sparse vectors [10] (that is also commonly perceived when applying the approach of structured imposing to audio sharing). follows:

An algorithm for the aforementioned steps is presented as

Algorithm: Structured Cosine Similarity

\section{Input:}

$x_{i}, i=1,2, \cdots, n:$ input documents

$p=0,1,2, \cdots, m:$ concept node number

$O$, the task-oriented ontology

Output:

$\operatorname{sim}\left(\overrightarrow{x_{a}}, \overrightarrow{x_{b}}\right) \quad$ measured similarity values,

$1 \leq a \leq n, 1 \leq b \leq n(a \neq b)$

Method:

(1) After the ASR processing of $x_{i}$, identify in $x_{i}$ the occurrences of the concept nodes $x_{i p}$ in the ontology.

(2) Translate $x_{i}$ with the approach of the s-txn imposing into a term vector $\vec{x}_{i}, \overrightarrow{x_{i}}=\left(x_{1 i}, x_{2 i}, \cdots, x_{m i}\right)$.

${ }^{5}$ This is because top-level concepts of words on the same path in the ontology have been accounted in the modified term frequencies of those top-level concepts. 
(3) Repeat step 1 and step 2 until all of the input documents are translated.

(4) Compute the similarity value between and two term vectors $\rightarrow \rightarrow$ $\left(x_{a}, x_{b}\right)$ with the measure of Cosine similarity: $\operatorname{sim}\left(\overrightarrow{x_{a}}, \overrightarrow{x_{b}}\right)=\frac{\vec{x}_{a}^{\mathrm{T}} \vec{x}_{b}}{\left\|\overrightarrow{x_{a}}\right\|_{2} \cdot\left\|\overrightarrow{x_{b}}\right\|_{2}}$.

(5) Repeat step 3 until the similarity values of all the pairs of documents are calculated.

The evidences of semantic accordance can be attained by asserting the underlying proximity semantic that the similarity value for nodes of the same path is smaller than that of nodes of sibling and that for nodes of the same path the similarity value of more distant nodes is smaller than that of less distant nodes [13].

\section{Evaluations}

With the proposed method of SCS document summarization, this section accommodates further evidences that show how SCS document summarization advances the SKC method of text clustering in terms of the clustering quality ${ }^{6}$.

The evaluations unfold as follows:

- Select a troubleshooting domain of certain electronics on which its task-oriented ontology can be constructed. This ontology is created based on a thorough examination of the FAQ site (of 554 FAQs) of an international renowned electronics company (as shown in Table1). The FAQs furnish us with appropriate troubleshooting knowledge in terms of the concepts and their troubleshooting causal relationships. The resulting ontology consists of 761 concept nodes spreading over 9 levels.

Table 1. The distribution of FAQs.

\begin{tabular}{|c|c|c|}
\hline Product & ID & Number of FAQs \\
\hline \hline $\begin{array}{c}\text { Black/White Laser } \\
\text { Printer }\end{array}$ & BL & 105 \\
\hline DigiCam & CA & 51 \\
\hline Color Laser Printer & CL & 74 \\
\hline Recorder & CR & 77 \\
\hline PDA & HA & 70 \\
\hline Notebook & NB & 43 \\
\hline Inkjet Printer & PR & 66 \\
\hline Scanner & SC & 68 \\
\hline
\end{tabular}

Note: An ID stands for a symbol (identifier) used to denote the designated product in the subsequent experiments.

- Decide the metrics used to measure the clustering quality for subsequent evaluations. Suppose the given $g$

\footnotetext{
${ }^{6}$ Please see [16] for the application of our method to audio based knowledge management (i.e., simply by voice mobile workforce of electronic troubleshooting are able to share and access troubleshooting experience).
}

categories $K_{h}, h \in\{1, \cdots, g\}$ of documents are dispersed into $k$ clusters $C_{l}, l \in\{1, \cdots, k\}$ with a document clustering method. Let $C^{*}$ represent the superset of the $k$ clusters, $n$ denote the total number of documents, $n_{l}$ and $n^{(h)}$ be the number of documents for the $l$-th cluster and the $h$-th category respectively, $n_{l}^{(h)}$ be the number of documents of the the $h$-th category in the $l$-th cluster. Three metrics (purity, entropy, normalized symmetric mutual information) are exerted and described as follows [10]:

- Purity:

$P\left(C_{l}\right)=\frac{1}{n_{l}} \max _{h}\left(n_{l}^{(h)}\right)$, the purity of a given cluster $C_{l}$ (i.e., the percentage of the major category of documents existing in the cluster).

The bigger purity $C_{l}$ has the more important role the major category plays in the cluster.

- Entropy:

$E\left(C_{l}\right)=-\frac{1}{\log (g)} \sum_{h=1}^{g} \frac{n_{l}^{(h)}}{n_{l}} \log \left(\frac{n_{l}^{(h)}}{n_{l}}\right)$, the degree of document categories scattering within a given cluster $C_{l}$.

The lower entropy $C_{l}$ has the more important role the major category plays in the cluster.

Normalized symmetric mutual information (NSMI):

$$
\begin{aligned}
\operatorname{NSMI}\left(C^{*}\right) & =\frac{2}{n} \sum_{l=1}^{k} \sum_{h=1}^{g} n_{l}^{(h)} \frac{\log \left(\frac{n_{l}^{(h)} / n}{n_{l} / n * n^{(h)} / n}\right)}{\log (k * g)} \\
& =\frac{2}{n} \sum_{l=1}^{k} \sum_{h=1}^{g} n_{l}^{(h)} \frac{\log \left(\frac{n_{l}^{(h)} * n}{n_{l} * n^{(h)}}\right)}{\log (k * g)}, \text { the }
\end{aligned}
$$
degree of document categories scattering among clusters.

NSMI outsmarts than purity and entropy when encountering the extreme situation of singleton clusters (i.e., a cluster of only 1 document) where purity and entropy equal to 1 and 0 respectively (even though this is an unfavorable clustering outcome). A higher NSMI value indicates a more desirable distribution of documents of various categories into clusters. Conduct the experiments and show the merits of SCS document summarization to the SKC method. In other words, we compare the performance of the SKC method with the three different methods of document summarization: the tfidf approach (originally employed in the SKC method and briefly named as the tfn approach), the structured imposing 
approach, and the s-txn approach. In order to gather comprehensive evaluation results, we conduct eight sets of experiments (in which myriad types of documents are solicited from the 554 FAQs in order to form different experiment conditions, each of which characterizes a situation of provisioned document space on which the SKC method is performed). As follows are the eight kinds of document spaces under evaluation:

(1) Documents of two categories easily differentiated but unevenly distributed (e.g., $\mathrm{BL}, \mathrm{NB}^{7}$ or $\mathrm{BL}, \mathrm{CA}$ are easily differentiated categories and thus randomly select from them the uneven amount of documents).

(2) Documents of two categories easily differentiated and evenly distributed.

(3) Documents of two categories not easily differentiated but evenly distributed (e.g., CL, PR or BL, CL are not easily differentiated categories).

(4) Documents of two categories not easily differentiated and unevenly distributed.

(5) Documents of the whole FAQs (that endow the document space of 8 categories or 6 categories when $\mathrm{BL}, \mathrm{CL}, \mathrm{PR}$ are united together as they are all about printers).

(6) Documents of the whole FAQs augmented with additional missing concepts (e.g, out of the 554 FAQs there are 423 FAQs that do not possess words appearing in the first and second level of concepts nodes of the ontology and thus can be further filled with the missing concepts in their corresponding speech documents).

(7) Documents of all of the categories that are evenly distributed (e.g, the document space consists of all of the FAQs (of which categories all sizing around 65 FAQs) except documents of CA (of size 51 FAQs) and of NB (of size 43 FAQs)).

(8) Documents of all of the easily differentiated categories (e.g., by removing those documents of categories not easily differentiated such as BL and CL for PR, the document space then consists of only documents of 6 categories).

For the document spaces of kinds (1)-(4) documents are selected randomly according to the designated experiment settings, while for the document spaces of kinds (5)-(8) all of the 554 documents are considered and tuned in accordance with the designated experiment settings.

Due to the limitation of space, we only summarize the evaluation results attained from the 8 kinds of experiments, accordingly manifesting the merits of SCS document summarization (for details, please see [13]):

- From Figure 3, with the documents of two categories not differentiated (regardless of their document amounts) we observe that the three approaches of document summarization perform around the same level of qualities

\footnotetext{
${ }^{7}$ Identifiers are addressed in Table 2.
}

(but the performance of s-txn is more stable with respect to the amounts of documents provisioned). However, the performance of structured and s-txn significantly outperforms tfn when given the documents of categories differentiated. Furthermore, the performance of tfn is the least stable with respect to the amounts of the documents.

- From Figure 4, when all of the documents are considered (and thus the number of categories considered increases), both structured and s-txn outperform tfn (except in the situation of all the documents evenly distributed where the three approaches perform around the same level of qualities). Furthermore, structured and s-txn benefit from the filling of the missing concepts (and thus behave semantics sound) while tfn does not.

- Figure 5 exhibits that structured behaves more like s-txn when considering all documents of categories more differentiated (in comparison with the original ones). However, s-txn exhibits a stabilized performance with respect to the increment added to the level of category differentiation.

- Figure 6 finally shows that tfn relatively is the least efficient approach in terms of the number of the unit operation (expectation maximization) required in the SKC method.

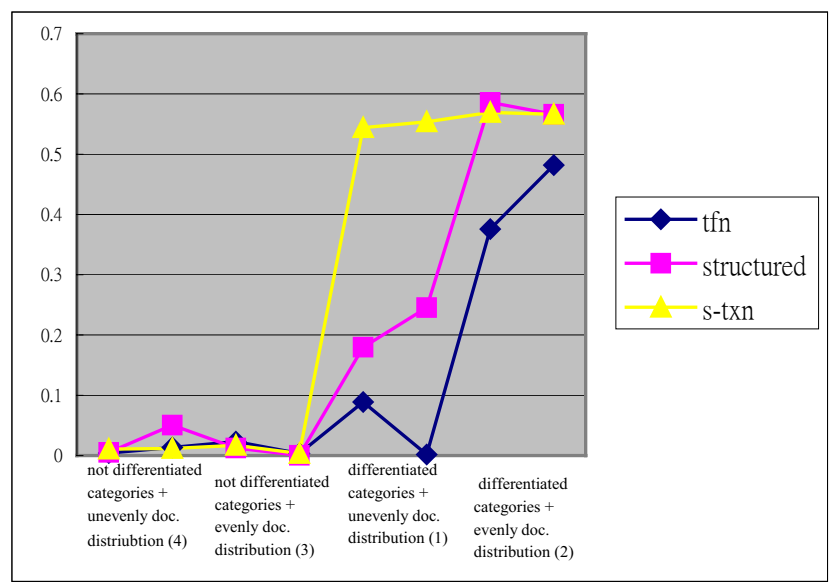

Figure 3. Summarized results for documents of two categories.

In short, SCS document summarization (structured or stxn), in general, outperforms tfn in terms of quality, stability and efficiency. Between structured and s-txn, s-txn then perform better than structured.

\section{Conclusion}

In this paper, we present a novel methodology named Structured Cosine Similarity (SCS) that furnishes text clustering with a new way of modeling on document summarization by considering the structure of terms in documents to improve the quality of document clustering. Furthermore, we equip the Spherical K-Means Clustering (SKC) method (a highly regarded method of document clustering due to its simplicity and good quality) with SCS, attaining evidences showing SCS bestows SKC a further advance in quality, stability and efficiency. This improvement is exceedingly important especially for the application of audio based knowledge management among mobile 
work force (owing to the limited resources provisioned for the handset devices of mobile work force). Future fruitful research includes the applicability of SCS to the other clustering methods (including the successors of the SKC method such as [15]) and the extension of the application domains with our implemented web services (in addition to the application of audio based knowledge management).

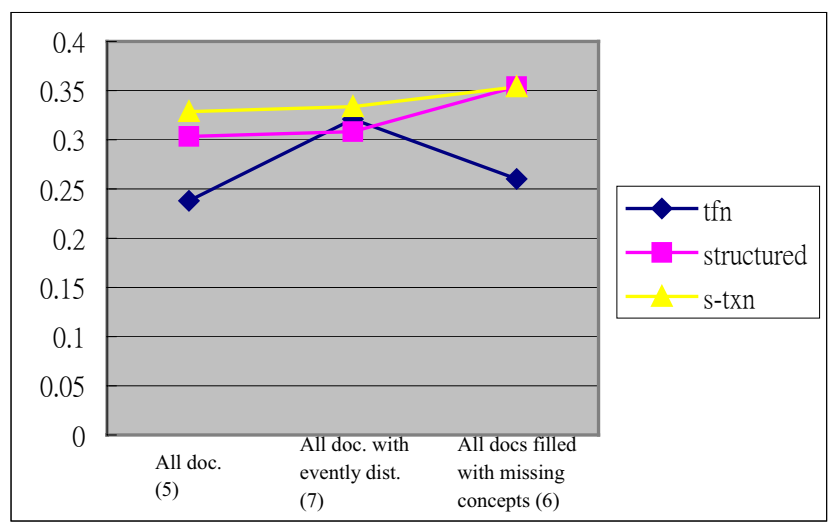

Figure 4. Summarized results for considering all of the documents regarding document distribution

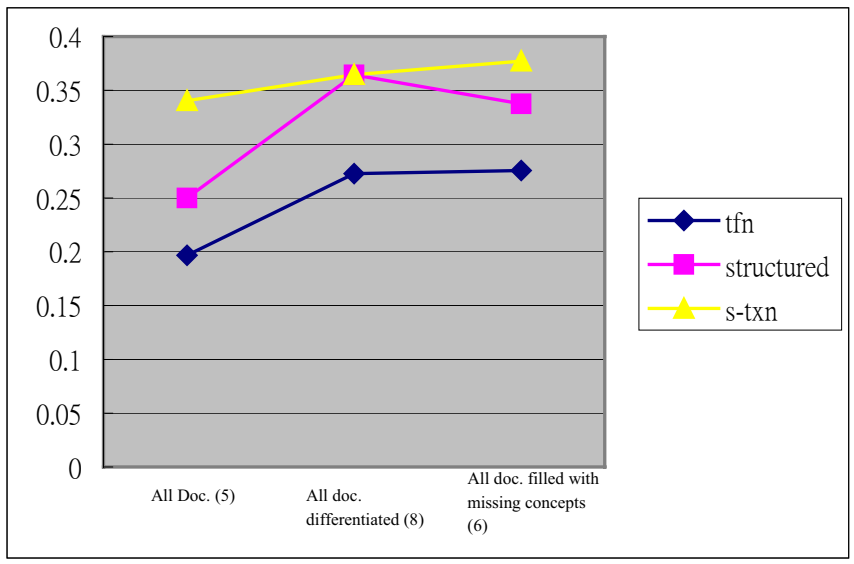

Figure 5. Summarized results for all of the documents regarding document differentiation.

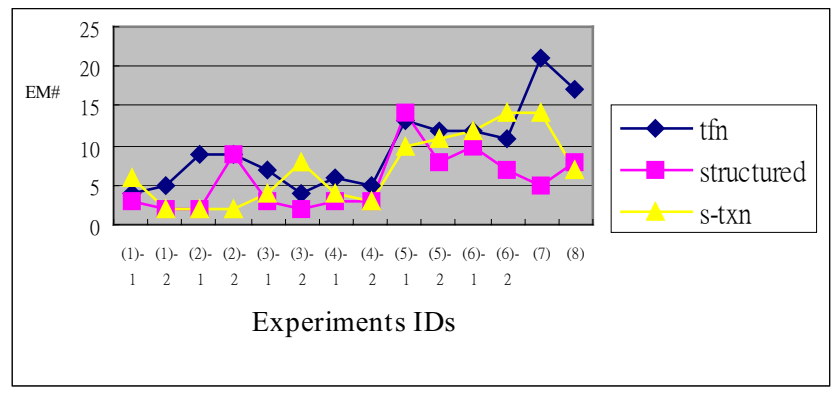

Figure 6. Summarized results regarding the efficiency of the three approaches.

\section{References}

[1] Hearst, Marti A. 1997. Text data mining: issues, techniques, and the relationship to information access. Presentation notes for UW/MS Workshop on Data Mining.

[2] Lang, K. 1995. NewsWeeder: learning to filter news, International Conference on Machine Learning. Tarragona, Spain.

[3] Deerwester, S., Dumais, ST, Furnas, GW, Landauer, TK, and Harshman, R. 1990. Indexing by latent semantic analysis. Journal of the American Society for Information Science, 41(6):391-407.

[4] Dumais, S. T. 1995. Using LSI for information filtering: TREC-3 experiments. The Third Text Retrieval Conference (TREC3), Gaithersburg, Maryland USA, pp.500-525.

[5] Littman, M. L. and Keim, G. A. 1997. Cross-language text retrieval with three Languages. International Conference of Neural Information Processing Systems. Breckenridge, Colorado, USA.

[6] Hong, Jason I. 2000. An overview of Latent Semantic Indexing, $\quad \mathrm{http}: / / \mathrm{www} . c s . b e r k e l e y . e d u /$ $\sim$ jasonh/classes/sims240/sims-240-final-paper-lsi_files/sims240-final-paper-lsi.doc.

[7] Dhillon, I.S., Fan, J., Guan, Y. 2001. Efficient clustering of very large document collections. In R. Grossman, G. Kamath, and R. Naburu, editors, Data Mining for Scientific and Engineering Applications, Kluwer Academic Publ.

[8] Dhillon, I. S. and Modha, D. S. 2001. Concept decompositions for large sparse text data using clustering, Machine Learning. 42(1): 143-175.

[9] Hartigan, J. A. and Wong, M. A. 1979. A K-means clustering algorithm. Applied Statistics. 28:100-108.

[10] Strehl, J. Ghosh and Mooney, R. 2000. Impact of similarity measures on web-page clustering. 7th National Conference on Artificial Intelligence: Workshop of Artificial Intelligence for Web Search, pp.58-64.

[11]IBM Chinese ViaVoice $\quad$ Telephony. http://www.research.ibm.com/beijing/projects/project1.html.

[12] Gruber, T. R. 1993. A translation approach to portable ontologies. Knowledge Acquisition, 5(2):199-220.

[13] Jerry Sun and Soe-Tsyr Yuan. 2003. Ontology-based taskoriented audio mining for mobile B2E applications. Tech. Report. Information Management Department, Fu-Jen University, Taiwan.

[14] Soe-Tsyr Yuan and K J. Lin. 2003. WISE - building simple intelligence into e-commerce. International Conference of Web Intelligence, Halifax, Canada.

[15] Jimenez, D., Enguix, CF, Vidal, V. 2002. A comparison of experiments with the Bisecting-Spherical K-Means clustering and SVD algorithms. Workshop on Processing and Information Retrieval. Polytechnic University of Valencia, Valencia, Spain. 